\title{
Variable expressivity of the clinical and biochemical phenotype associated with the apolipoprotein $E$ p.Leu149del mutation
}

\author{
Laurence Faivre ${ }^{*, 1}$, Pascale Saugier-Veber ${ }^{2}$, Jean-Paul Pais de Barros ${ }^{3}$, Bruno Verges ${ }^{4}$, \\ Bertrand Couret $^{5}$, Bernard Lorcerie ${ }^{6}$, Christel Thauvin ${ }^{1}$, Françoise Charbonnier ${ }^{2}$, \\ Frédéric Huet ${ }^{1}$, Philippe Gambert ${ }^{3}$, Thierry Frebourg ${ }^{2}$ and Laurence Duvillard ${ }^{3}$
}

${ }^{1}$ Centre de Génétique Médicale, Hôpital d'Enfants, Dijon, France; ${ }^{2}$ Laboratoire de Génétique Moléculaire et INSERM U614, Faculté de Médecine et de Pharmacie, Service de Génétique, Rouen, France; ${ }^{3}$ INSERM U498, Faculté de Médecine, Dijon, France; ${ }^{4}$ Endocrinologie, Centre Hospitalier le Bocage, Dijon, France; ${ }^{5}$ Service de Médecine Interne, Hôpital Purpan, Toulouse, France; ${ }^{6}$ Service de Médecine Interne, Centre Hospitalier le Bocage, Dijon, France

Splenomegaly with sea-blue histiocytes, thrombocytopenia and hypertriglyceridemia is a very rare association that has been described in only one report to date. The molecular defect in the two reported patients consists in a deletion of a leucine at position 149 in the receptor-binding region of the apoE molecule. Here, we report on another family in whom the proband and his brother were diagnosed with splenomegaly, thrombocytopenia and hypertriglyceridemia. An apoE p.Leu149del mutation was found in both subjects. A large $\beta$ band in the VLDL fraction and elevated VLDL cholesterol-to-plasma triglyceride ratio was observed in the proband only. Their mother, presenting with isolated hypertriglyceridemia, also carried the same p.Leu149del mutation. The coexistence of factors facilitating the development of hypertriglyceridemia and/or low HDL-cholesterol level could explain why the proband and his brother developed a splenomegaly with thrombocytopenia, whereas the mother did not. Moreover, the presence of an apoE2 allele in the proband likely explains the more severe phenotype we observed in this subject. In conclusion, the apoE p.Leu149del mutation results in a very striking phenotype including one or all symptoms among splenomegaly, thrombocytopenia and hypertriglyceridemia, and should be considered as a differential diagnosis of storage disorders in the causes of splenomegaly with sea-blue histiocytes. European Journal of Human Genetics (2005) 13, 1186-1191. doi:10.1038/sj.ejhg.5201480;

published online 10 August 2005

Keywords: hypertriglyceridemia; splenomegaly; thrombocytopenia; apolipoprotein E

\section{Introduction}

Splenomegaly with sea-blue histiocytes is a rare feature characterized by the presence of numerous histiocytes containing cytoplasmic granules which stain bright blue with usual hematologic stains. ${ }^{1}$ This type of splenomegaly

*Correspondence: Dr L Faivre, Centre de Génétique, Hôpital d'Enfants, 10, bd Maréchal DeLattre de Tassigny, 21034 Dijon, France.

Tel: + 33380293 300; Fax: + 33380293 266;

E-mail: laurence.faivre@chu-dijon.fr

Received 1 December 2004; revised 8 April 2005; accepted 30 June 2005; published online 10 August 2005 has been described in several conditions, including cholesterol ester storage disease and other lysosomal disorders (Niemann-Pick, Gaucher diseases), severe hypertriglyceridemia, lecithin-cholesterol acyltransferase deficiency and Tangier diseases. ${ }^{2}$ In two patients, splenomegaly with sea-blue histiocytes was shown to be associated with thrombocytopenia and mild hypertriglyceridemia, and to result from a $3 \mathrm{bp}$ deletion of the $A P O E$ gene. This in-frame deletion induces the loss of the leucine residue at position 149 within the receptor-binding region of the apoE. ${ }^{3}$ As shown in vitro, this apoE mutant stimulates cholesterol 
ester accumulation and the targeting of lipoproteins to macrophages, resulting in splenomegaly. These results were supported by the observation that splenectomy in both probands induced an increase in plasma lipid level and therefore by the accumulation of lipoprotein remnants in the intravascular compartment. Here, we report on another family presenting the association of splenomegaly with sea-blue histiocytes, thrombocytopenia and hypertriglyceridemia with a similar deletion of leucine at position 149 of the APOE gene, and give further delineation of the disease.

\section{Materials and methods Patients}

Our proband II-4, a 47-year-old Caucasian male, was referred to the genetic clinics for suspicion of storage disease because of the association of thrombocytopenia, splenomegaly with sea-blue histiocytes and similar clinical features in one of his brothers (Figure 1). Our patient was known to have several cardiovascular risk factors, including vascular hypertension, diabetes mellitus, hypertriglyceridemia and hypercholesterolemia with unsatisfactory responses to various medical treatments (fenofibrate, atorvastatin, gemfibrozil, pravastatin), tabagism and obesity (body mass index $=30 \mathrm{~kg} / \mathrm{m}^{-2}$ ). At 42 years of age, he had a noticeable thrombocytopemia on routine examination $\left(76000 / \mathrm{mm}^{3}\right)$ with normal platelet shape. Triglyceride levels were markedly elevated $(13.6 \mathrm{mmol} / \mathrm{l}, N<1.50)$ with opalescent serum, cholesterol level was normal $(4.8 \mathrm{mmol} /$ 1, $N<6)$. Aspartate aminotransferase $(46 \mathrm{UI} / \mathrm{l}, \mathrm{N}<37)$ and alanine aminotransferase $(79 \mathrm{UI} / \mathrm{l}, N<40)$ were slightly elevated. Clinical examination revealed a splenomegaly and the common causes of splenomegaly, including infection and malignancy, could be excluded. There was no xanthoma and ophtalmological examination was normal. The association of unexplained stable thrombocytopenia and splenomegaly led, 1 year later, to splenectomy. Spleen measured $20 \times 12 \times 7 \mathrm{~cm}$ and weighed $810 \mathrm{~g}$. Anatomopathological examination revealed numerous splenic infarctions and showed that the red pulp was markedly expanded by foamy histiocytes, consistent with sea-blue histiocytes. The white pulp was hypoplastic. Similar foamy histiocytes were observed in the bone marrow. A NiemannPick type B or C was initially suspected but leukocyte sphingomyelinase levels and cholesterol esterification in cultured fibroblasts were in the normal range. Follow-up showed the rapid normalization of platelet counts. In contrast, triglyceride levels worsened ranging from 3.03 to $28.4 \mathrm{mmol} / \mathrm{l}$, depending on the medical treatment of the patient, and total cholesterol levels also significantly increased (5.1-14.8 mmol/l). Cardiac investigations, including an electrocardiogram and an exercise test, were normal.

Family history revealed mild hypercholesterolemia and hypertriglyceridemia in the proband's father I-2 with severe documented ischemic heart disease (Figure 1). His first cardiac alert was noted at 39 years of age and he later presented myocardial infarction. A pacemaker was indicated for cardiac rhythm abnormalities and he had medical treatment for hypothyroidism. The proband's mother I-1 (Figure 1) also presented with a past medical history of hypertriglyceridemia ranging from 3 to $4 \mathrm{mmol} / \mathrm{l}$, with satisfactory control under atorvastatin treatment. She also had medical treatment for hypothyroidism and Parkinson disease. Clinical and ultrasound examination of the spleen was normal as well as platelet counts in both parents. The proband also had two brothers, and only one of them

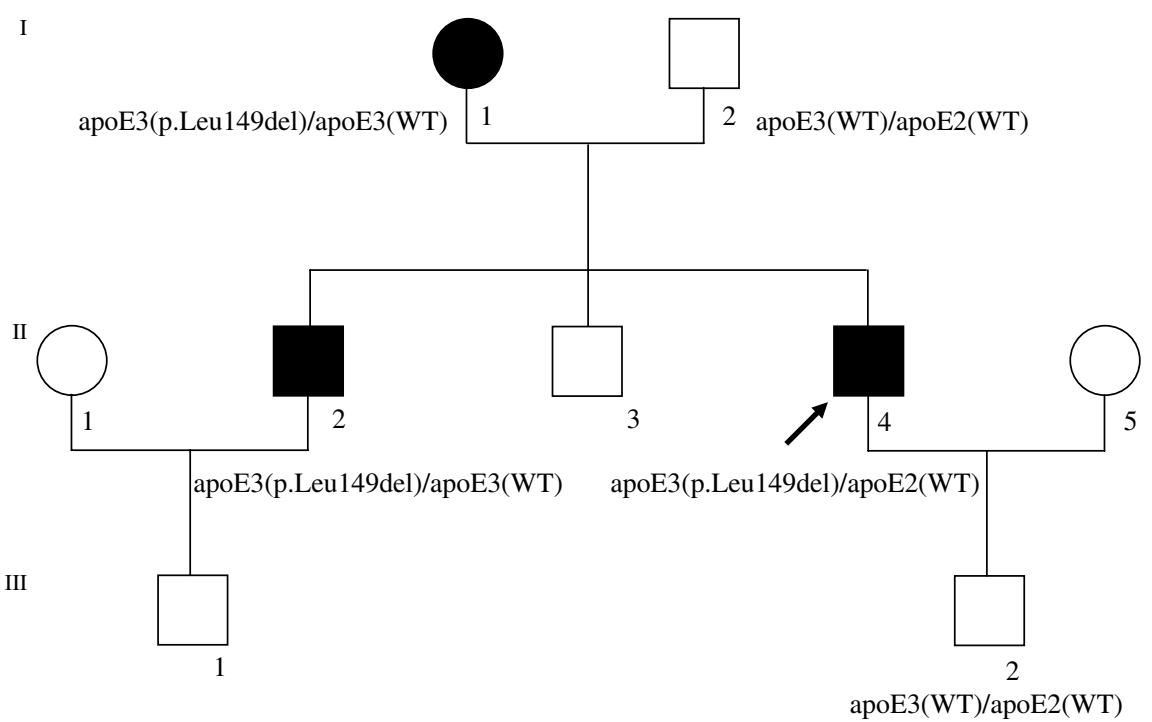

Figure 1 Pedigree and $A P O E$ genotypes in the present reported family. 
presented a noticeable medical history (II-2, Figure 1). He had clinical and biological follow-up for moderate hypertriglyceridemia and thrombocytopenia $\left(100000 / \mathrm{mm}^{3}\right)$, splenomegaly $(17.5 \times 9 \times 5.5 \mathrm{~cm})$ and ischemic heart disease documented since 47 years of age. Triglycerides ranged from normal to $4.30 \mathrm{mmol} / \mathrm{l}$, total cholesterol was normal to slightly elevated (up to $6.8 \mathrm{mmol} / \mathrm{l}$ ) and aspartate aminotransferase was also elevated (156 UI/l). He had been treated by gemfibrozil, later replaced by atorvastatin but the hypolipemic treatment was stopped because of severe myolysis. Both our proband and his affected brother have a child with noticeable learning difficulties.

\section{Plasma lipid and lipoprotein analysis}

Total and HDL-cholesterol, triglycerides, protein and apoB were measured on a Dimension analyser with dedicated reagents (Dade Behring, Newark, DE, USA). LDL-cholesterol was calculated using the Friedewald formula. Apolipoprotein E was measured by turbidimetry on a Dimension analyser with reagents purchased by ImmunoChimie International (Bouffémont, France). Free cholesterol and phospholipids were measured on the same analyser with Wako reagents (Neuss, Germany).

Very low density $(d)$ lipoproteins (VLDL) $(d<1.006 \mathrm{~g} / \mathrm{ml})$, intermediate density lipoproteins (IDL) $(1.006<d<1.019$ $\mathrm{g} / \mathrm{ml})$, low density lipoproteins (LDL) $(1.019<d<1.063$ $\mathrm{g} / \mathrm{ml})$ and high density lipoproteins (HDL) $(1.063<d<1.21$ $\mathrm{g} / \mathrm{ml}$ ) were isolated from serum by sequential flotation ultracentrifugation using a 50.4 rotor in an L7 apparatus (Beckman Instruments, Palo Alto, US). The VLDL cholesterolto-plasma triglyceride ratio was calculated: this ratio suggests an accumulation of VLDL remnants when above $0.3 .{ }^{4}$

Agarose gel electrophoresis was performed using the Paragon LIPO Gel kit (Beckman Coulter) and scanned with Gel doc 2000 (Bio-Rad, Richmond, CA, USA). Serum lipoproteins were fractionated by fast protein liquid chromatography (FPLC) on a Superose 6HR 10/30 gel filtration column (Amersham Pharmacia Biotech). Lipoproteins were eluted at a constant flow rate of $0.3 \mathrm{ml} / \mathrm{min}$ with TSE buffer $(50 \mathrm{~mm}$ Tris, $0.15 \mathrm{M} \mathrm{NaCl}, 1 \mathrm{~mm}$ EDTA, $0.02 \% \mathrm{NaN}_{3}, \mathrm{pH} 7.4$ ). Forty six $0.3 \mathrm{ml}$ fractions were collected. VLDL, IDL + LDL and HDL were eluted in fractions of 8-16, 17-30 and 31-46 ml, respectively. Total cholesterol was assayed in each individual fraction on a Victor 1420 Multilabel Counter (Wallac), using Cholesterol 100 reagent (ABX Diagnostics, Montpellier, France).

\section{Molecular analyses}

Blood samples were obtained after written informed consent for each patient. Genomic DNA was extracted from peripheral blood lymphocytes using QIAamp DNA blood mini kit (Qiagen, Courtaboeuf, France). The three coding exons of the APOE gene were PCR-amplified using intronic primers for exons 2 and 3 and using two sets of primers for exon 4 which is $859 \mathrm{bp}$ long (Table 1). PCR reactions were performed in a final volume of $50 \mu \mathrm{l}$ containing $0.5 \mu \mathrm{mol} / 1$ of each primer and $1 \mathrm{U}$ Taq DNA polymerase (Abgene, Courtaboeuf, France). After an initial denaturation of $3 \mathrm{~min}$ at $95^{\circ} \mathrm{C}$, the PCR consisted of 12 cycles of $15 \mathrm{~s}$ at $94^{\circ} \mathrm{C}, 15 \mathrm{~s}$ at $60^{\circ} \mathrm{C}$ (with a decrease of $1^{\circ} \mathrm{C}$ per cycle) and $30 \mathrm{~s}$ at $72^{\circ} \mathrm{C}$, then 35 cycles of $10 \mathrm{~s}$ at $94^{\circ} \mathrm{C}$, $15 \mathrm{~s}$ at $48^{\circ} \mathrm{C}$ and $30 \mathrm{~s}$ at $72^{\circ} \mathrm{C}$ and was followed by a final extension of $5 \mathrm{~min}$ at $72^{\circ} \mathrm{C}$. PCR products were purified using the Qiaquick Gel Extraction kit (Qiagen, Courtaboeuf, France) after electrophoresis on a $2 \%$ Seakem LE agarose (FMC, Rockland, ME, USA) gel. Sequencing reactions were performed using the Big-dye Terminator v3.1 cycle Sequencing kit (Applied Biosystems, Courtaboeuf, France) and sequences were analyzed on an ABI 377automated DNA sequencer (Applied Biosystems, Courtaboeuf, France). Screening for the c.499_501delCTC mutation was performed by fragment length analysis on an ABI3100-automated sequencer (Applied Biosystems, Courtaboeuf, France). A portion of exon 3 of the APOE gene including the in-frame deletion was PCR-amplified using the following primers: 5'-6-FamGCCTACAAATCGGA ACTGGA-3' and 5'-GCGCTTCTGCAGGTCATC-3'. In all, $1 \mu \mathrm{l}$ of the PCR product was loaded on the ABI3100 DNA sequencer. Data were analyzed using the ABI PRISM ${ }^{\circledR}$ GeneScan ${ }^{\circledR}$ Analysis Software version 3.7.1 (Applied Biosystems, Courtaboeuf, France).

\begin{abstract}
Results
Lipid parameters are presented in Table 2 for each subject. Plasma triglycerides and total cholesterol were high in the proband, whereas HDL-cholesterol was moderately low. In the brother, we observed a moderate hypertriglyceridemia and a decreased HDL-cholesterol level. In the mother, plasma triglycerides were in the normal range, whereas LDL-cholesterol was slightly elevated. The father presented hypertriglyceridemia and a moderately low HDL-cholester-
\end{abstract}

Table 1 Primers used for the sequencing of the entire coding region of the APOE gene

\begin{tabular}{|c|c|c|c|}
\hline Exon & Sense primer & Antisense primer & Size of the amplicon $(b p)$ \\
\hline $\begin{array}{l}\text { Exon } 2 \\
\text { Exon } 3 \\
\text { Exon } 4-1 \\
\text { Exon } 4-2\end{array}$ & 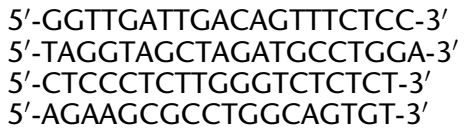 & 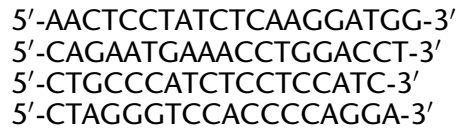 & $\begin{array}{l}278 \\
332 \\
591 \\
548\end{array}$ \\
\hline
\end{tabular}


Table 2 Fasting lipid parameters in the proband and his family members

\begin{tabular}{|c|c|c|c|c|}
\hline APOE genotype & $\begin{array}{c}\text { Proband }(I I-4)^{\mathrm{a}, \mathrm{b}} \\
\text { p.Leu149del/WT } \\
\text { E3/E2 }\end{array}$ & $\begin{array}{c}\text { Brother }(I 1-2)^{\mathrm{a}} \\
\text { p.Leu149del/WT } \\
\text { E3/E3 }\end{array}$ & $\begin{array}{c}\text { Mother }(I-1)^{\mathrm{a}} \\
\text { p.Leu149del/WT } \\
\text { E3/E3 }\end{array}$ & $\begin{array}{c}\text { Father (I-2) } \\
\text { WT/WT } \\
\text { E3/E2 }\end{array}$ \\
\hline $\begin{array}{l}\text { Plasma triglycerides }(\mathrm{mmol} / \mathrm{l}) \\
\text { Plasma total cholesterol }(\mathrm{mmol} / \mathrm{l}) \\
\text { Plasma HDL-cholesterol }(\mathrm{mmol} / \mathrm{l}) \\
\text { LDL-cholesterol }(\mathrm{mmol} / \mathrm{l}) \\
\text { VLDL-cholesterol/plasma triglycerides } \\
\text { Plasma apoB }(\mathrm{g} / \mathrm{l}) \\
\text { Plasma apoE }(\mathrm{mg} / \mathrm{l})\end{array}$ & $\begin{array}{c}4.31 \\
9.33 \\
1.02 \\
\mathrm{NC} \\
0.75 \\
1.68 \\
148.00\end{array}$ & $\begin{array}{r}1.87 \\
5.68 \\
0.67 \\
4.16 \\
0.22 \\
1.71 \\
58.00\end{array}$ & $\begin{array}{r}0.96 \\
6.53 \\
1.52 \\
4.57 \\
0.15 \\
1.62 \\
41.00\end{array}$ & $\begin{array}{r}2.60 \\
5.57 \\
1.00 \\
3.39 \\
0.20 \\
1.20 \\
93.00\end{array}$ \\
\hline
\end{tabular}

${ }^{a}$ At the time of study, the proband was treated by pravastatin, the mother by atorvastatin and the brother was untreated.

${ }^{\mathrm{b}}$ Fasting lipid parameters postsplenectomy.

WT: wild type; NC: could not be calculated.

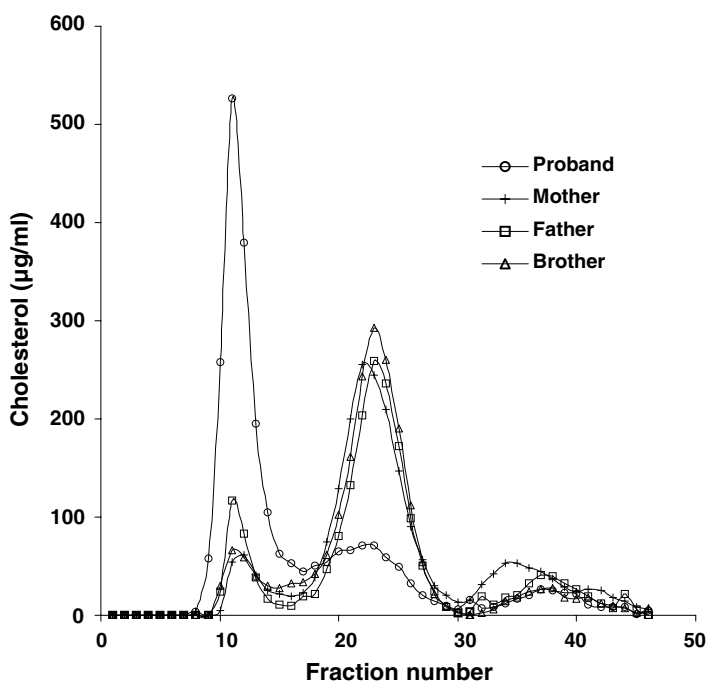

Figure 2 Cholesterol distribution in serum lipoproteins from the four studied subjects. Serum lipoproteins were fractionated by fast protein liquid chromatography (FPLC) on a Superose 6HR 10/30 gel and the cholesterol content of the different fractions was determined as detailed in Materials and methods.

ol level. The FPLC cholesterol profile (Figure 2) showed that the distribution of cholesterol in the proband was totally different from that observed in the other members of the family. Indeed, in the proband, 66, 25 and 9\% of serum cholesterol was eluted in VLDL, IDL + LDL and HDL, respectively. In the mother, the proportions were 10, 69 and $21 \%$. In the father, they were 15,69 and $16 \%$ and in the brother 14, 76 and $10 \%$. Moreover, the VLDL cholesterol-to-plasma triglyceride ratio was 0.75 in the proband, which characterizes the accumulation of VLDL remnants in the intravascular compartment. In contrast, it was below 0.3 in the other members of the family. In the proband, agarose gel electrophoresis showed a large $\beta$ band in the VLDL fraction, characteristic of VLDL remnants (Figure 3). In his brother, such a band could also be

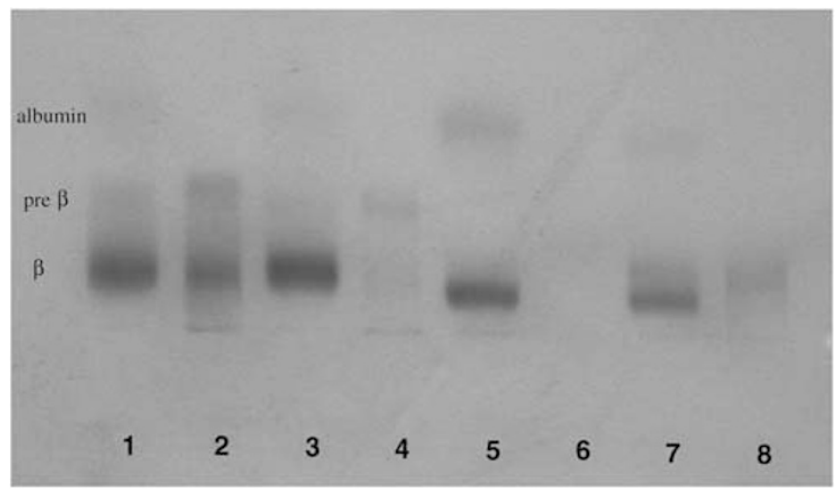

Figure 3 Agarose gel electrophoresis of serum and VLDL fraction in the different members of the family. Lines 1 and 2: Proband's serum and VLDL, respectively (II-4); lines 3 and 4: brother's serum and VLDL (II-2); lines 5 and 6: mother's serum and VLDL (I-1): lines 7 and 8: father's serum and VLDL (I-2).

observed, although it was much less pronounced. Neither the mother nor the father exhibited a typical $\beta$ band in the VLDL fraction. The analysis of lipoprotein composition also argues for the accumulation of VLDL remnants in the proband. Indeed, in the proband, VLDL were more cholesteryl ester and less triglyceride rich than in the other family members (Table 3 ).

The sequencing of the entire coding region of the APOE gene in the index case II-4 led to the identification of a heterozygous deletion, c.499_501delCTC, in exon 4 of the APOE gene, which predicts p.Leu167del in the preprocessed peptide and p.Leu149del in the processed peptide (Figure 4). This c.499_501delCTC variation was not detected in 100 control chromosomes, indicating that this mutation did not correspond to a frequent polymorphism (data not shown). This in-frame mutation, which has been previously described in the context of a sea-blue histiocytes syndrome, ${ }^{3}$ was then identified in his brother II- 2 and his mother I-1. In contrast, neither his son III-2 nor his father 
Table 3 Composition of the different subclasses of lipoproteins for the four subjects studied

\begin{tabular}{|c|c|c|c|c|}
\hline & Proband (II-4) & Brother (II-2) & Mother (I-1) & Father (I-2) \\
\hline \multicolumn{5}{|l|}{$V L D L$} \\
\hline Proteins (\%) & 5.3 & 5.3 & 4.8 & 4.9 \\
\hline Phospholipids (\%) & 18.2 & 10.3 & 12.9 & 13.4 \\
\hline Free cholesterol (\%) & 10.7 & 6.2 & 4.8 & 1.9 \\
\hline Esterified cholesterol (\%) & 18.5 & 11.5 & 6.5 & 7.9 \\
\hline Triglycerides (\%) & 47.3 & 66.7 & 71.0 & 68.9 \\
\hline \multicolumn{5}{|l|}{$I D L$} \\
\hline Proteins (\%) & 9.4 & 10.1 & 9.9 & 10.8 \\
\hline Phospholipids (\%) & 20.3 & 22.2 & 21.4 & 22.9 \\
\hline Free cholesterol (\%) & 10.3 & 9.2 & 9.1 & 8.8 \\
\hline Esterified cholesterol (\%) & 36.8 & 36.9 & 39.1 & 38.5 \\
\hline Triglycerides (\%) & 23.2 & 21.6 & 20.5 & 19.0 \\
\hline \multicolumn{5}{|l|}{$L D L$} \\
\hline Proteins (\%) & 18.5 & 16.0 & 14.9 & 16.1 \\
\hline Phospholipids (\%) & 20.2 & 26.7 & 27.1 & 25.9 \\
\hline Free cholesterol (\%) & 8.1 & 9.3 & 9.8 & 8.3 \\
\hline Esterified cholesterol (\%) & 36.1 & 39.6 & 41.0 & 41.4 \\
\hline Triglycerides (\%) & 17.1 & 8.3 & 7.2 & 8.3 \\
\hline \multicolumn{5}{|l|}{$H D L$} \\
\hline Proteins (\%) & 45.9 & 49.7 & 44.0 & 47.0 \\
\hline Phospholipids (\%) & 24.6 & 22.8 & 26.4 & 25.2 \\
\hline Free cholesterol (\%) & 3.6 & 3.4 & 4.1 & 3.3 \\
\hline Esterified cholesterol (\%) & 17.7 & 20.1 & 22.9 & 21.7 \\
\hline Triglycerides (\%) & 8.2 & 4.0 & 2.6 & 2.8 \\
\hline
\end{tabular}

Results are expressed in percentage of total lipoprotein mass.

I-2 shared the mutation (Figure 1). Furthermore, the sequencing analysis allowed the determination of the $A P O E$ allele carrying the mutation. Indeed, the presence of the homozygous c.334T variation (p.Cys112) and of a heterozygous c.472C $>\mathrm{T}$ variation (p.Arg158Cys substitution) in the index case II2 determined his apoE3/apoE2 status. The analysis of the cosegregation of the mutation and the apoE isoforms in the family led to the conclusion that the p.Leu149del deletion was carried by an apoE3 allele (Figure 1).

\section{Discussion}

Nguyen et $a l^{3}$ have previously demonstrated that the mutant apoE3 p.Leu149del binds lipoproteins with high affinity to macrophages, which explains the development of splenomegaly with sea-blue histiocytes in subjects carrying this mutation, despite the lack of severe dyslipidemia. Here, we report on a family with the same apoE mutation (p.Leu149del) and give further delineation of clinical and biochemical phenotype in affected patients. The occurrence of the mutation in a French family gives further evidence in favor of a French founder effect ancestry of the APOE deletion (p.Leu149del) as discussed in the previous publication. ${ }^{3}$ The age of onset of symptoms

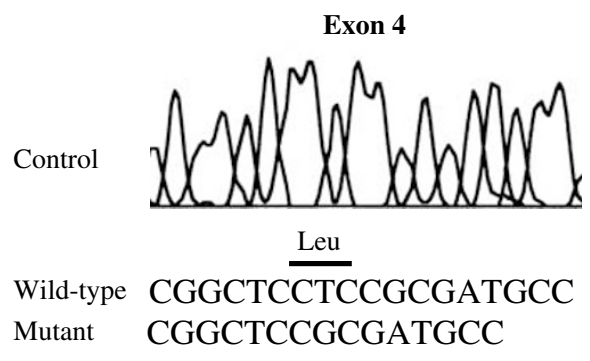

II-4

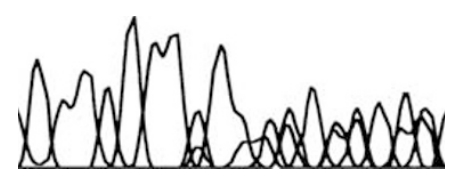

Figure 4 Identification of the $A P O E$ p.Leu149del (c.499_501delCTC) mutation by sequencing analysis. Patient II-4 is heterozygous for this in-frame deletion.

remains unknown, but all patients were reported between the second and the fifth decade. All patients with the full phenotype (hypertriglyceridemia, splenomegaly and thrombocytopenia) are males, with variable expression of the disease. The occurrence of elevated plasma liver transaminases in our patients as well as in the previous report suggests that targeting is not specific to splenic 
macrophages and that these features can be part of the diagnosis criteria.

Differential apoE isoform in the patient (apoE3/apoE2) and his brother (apoE3/apoE3) as well as in the previous reported patients (apoE3/apoE3) could be one explanation for the severity of hypertriglyceridemia in the proband. There are three common isoforms for apoE: E2, E3 and E4. Whereas apoE3 is considered to be the wild-type form, apoE2 (p.Arg158Cys) is defective in receptor binding, which leads to accumulation of $\beta$-VLDL in the intravascular compartment. ${ }^{5}$ The role of a single apoE2 allele in the worsening of hypertriglyceridemia in the proband is likely. Nguyen et $a l^{3}$ demonstrated that the VLDL from a subject with an mutant apoE3 allele (p.Leu149del) and a normal apoE3 allele bound normally to the LDL receptor. However, we cannot exclude that this normal binding was mainly due to the nonmutated allele. Usually, a single apoE2 allele does not induce hyperlipidemia. The presence of type III hyperlipidemia in our proband suggests that the mutant apoE3 allele (p.Leu149del) is not able to ensure a normal VLDL and chylomicron clearance. The defect can be either due to a defect binding of mutant apoE3 (p.Leu149del) to LDL receptors or to a decreased mutant apoE3 (p.Leu149del) affinity to proteoglycans. In our patient, the association of an E2 allele with a mutant E3 allele (p.Leu149del) may decrease the catabolic rate of remnants and worsen hypertriglyceridemia.

LDL-cholesterol concentration was lower in the proband, compared to the other members of the family. The proband was under pravastatin, which induces the expression of LDL receptor and thus an acceleration of LDL catabolism. On the other hand, the decrease uptake of VLDL remnants by the LDL receptor is likely to decrease the amount of cholesterol to the cells, which in turn induces an overexpression of LDL receptor in order to control the amount of cholesterol uptake by cells.

We wondered why the proband and his brother developed a splenomegaly, whereas the mother did not. A family history of hypertriglyceridemia in the father's side, although of different cause, may have contributed to a more severe expression of the apoE deletion symptomatology in the two brothers compared to their mother. The proband and his brother were found to have a metabolic syndrome, a disorder characterized by hypertriglyceridaemia and an increased number of secreted VLDL particles. Such a hypertriglyceridaemia may have facilitated the uptake and accumulation of VLDL in spleen macrophages, whereas the mother may have been protected from the development of splenomegaly because of a small number of circulating VLDL particles. On the other hand, HDLcholesterol was much lower in the proband and his brother than in their mother. So, the lower HDL-cholesterol level observed both in the proband and in his brother may be related to a less efficient reverse cholesterol transport, which facilitates the accumulation of cholesterol in spleen macrophages and the development of splenomegaly. ${ }^{6,7}$ Finally, it has also been shown that type III hyperlipidemia was much more common in men and tends to occur earlier in men than in women. ${ }^{5}$

In conclusion, this report gives further emphasis that, besides to storage disease, the 149 variant of apoE appears as a cause of splenomegaly with sea-blue histiocytes, when associated to hypertriglyceridemia, thrombocytopenia. Variable expressivity is striking and some family members, especially women, might present with mild hypertriglyceridemia only.

\section{References}

1 Silverstein MN, Ellefson RD, Ahern EJ: The syndrome of the seablue histiocyte. N Engl J Med 1970; 282: 1-4.

2 Silverstein MN, Ellefson RD: The syndrome of the sea-blue histiocyte. Semin Hematol 1972; 9: 293-307.

3 Nguyen TT, Kruckeberg KE, O'Brien JF et al: Familial splenomegaly: macrophage hypercatabolism of lipoproteins associated with apolipoprotein E mutation (apolipoprotein E (delta149 Leu)). J Clin Endocrinol Metab 2000; 85: 4354-4358.

4 Mahley RW: Apolipoprotein E: cholesterol transport protein with expanding role in cell biology. Science 1988; 240: 622-630.

5 Mahley RW, Rall SC: Type III hyperlipoproteinemia; in Betteridge DJ, Illingworth DR, Sheperd J (eds): Lipoproteins in Health and Diseases. New York: Arnold, 1999, pp 719-736.

6 Shah PK, Kaul S, Nilsonn J, Cercek B: Exploiting the vascular protective effects of high - density lipoprotein and its apolipoproteins. Circulation 2001; 104: 2376-2383.

7 Hurst RT, Lee RW: Increased incidence of coronary atherosclerosis in type 2 diabetes mellitus: mechanisms and management. Ann Intern Med 2003; 139: 824-834. 